\title{
Pectus excavatum repair from a plastic surgeon's perspective
}

\author{
Anton H. Schwabegger \\ Clinical Department of Plastic, Reconstructive and Aesthetic Surgery, Medical University of Innsbruck, Innsbruck, Austria \\ Correspondence to: Anton H. Schwabegger, MD, MSc, Assoc. Professor. Clinical Department of Plastic, Reconstructive and Aesthetic Surgery, \\ Medical University of Innsbruck, Anichstrasse 35, 6020 Innsbruck, Austria. Email: anton.schwabegger@i-med.ac.at.
}

\begin{abstract}
Minimally invasive repair of pectus excavatum (MIRPE) or similar procedures for pectus excavatum (PE) repair, nowadays no longer performed by one single speciality, may not always achieve sufficient aesthetic results, particularly in the infrapectoral or infraxiphoidal region. Reasons for this include the diaphragm inhibiting correct positioning of the bars, as well as asymmetric deformities which may still be present after remodelling attempts. Furthermore, some cases develop a mild recurrence or partial concavity once the correction bar is removed. However, any secondary re-do MIRPE procedure remains risky because of adhesions between the pleura, lung, pericardium, thoracic wall as residuals from the primary intervention. Treatment options as secondary correction for these deformities may include open access surgery, resection or reshaping of deformed costal cartilage. Moreover, augmentation of a residual concave area can be achieved by autologous transplantation of resected over-abundant cartilage, as well as by liposhifting or implantation of customized alloplastics. A physician dealing with PE corrections should be familiar with various shaping and complementary reconstructive techniques in order to provide the best options for a variety of expressions of anterior wall deformities. Among treating surgeons, there is an awareness that no single method can be applied for every kind of funnel chest deformity. An appropriate technique, either as a single approach for the ordinary deformities or in conjunction with ancillary procedures for the intricate cases, should be selected carefully based on the heterogeneity of symptoms, severity, expectations and surgical skill in addition to the available equipment. Out of a variety of such ancillary procedures available and based on experience within general plastic reconstructive surgery, some techniques for PE repair are explained and illustrated here with their advantages and disadvantages.
\end{abstract}

Keywords: Pectus excavatum (PE); silastic; flaps; autologous tissue

Submitted Mar 23, 2016. Accepted for publication Aug 08, 2016.

doi: 10.21037/acs.2016.09.01

View this article at: http://dx.doi.org/10.21037/acs.2016.09.01

\section{Introduction}

Plastic surgeons familiar with the technical scope of pectus excavatum (PE) repair utilizing the Ravitch technique, minimally invasive repair of pectus excavatum (MIRPE), modified open videoendoscopically assisted repair of pectus excavatum (MOVARPE) or other variants with or without hybridization of methods are still an exception rather than the rule within the society of surgeons $(1,2)$. While prior to the advent of MIRPE, the correction and remodelling of $\mathrm{PE}$ with an open approach was deemed a quite invasive procedure, it was reserved for severe cases with significant cardiopulmonary restrictions. Then and now, the majority of adolescent or adult patients with congenital PE predominantly suffer from disturbing aesthetics, so that plastic surgeons have been increasingly challenged to develop methods and technical refinements. They are specifically asked to correct the non-symptomatic $\mathrm{PE}$ with minimal invasiveness and with particular respect to a cosmetically desired result.

Previously, more than five decades ago, the domain of plastic surgery was restricted to extrathoracic corrections using self-vulcanizing silastic implants placed in a subcutaneous presternal pocket $(3,4)$. Nowadays, with improved quality of customized implantable silastic devices, this technique remains attractive as an alternative or even 
an adjunct to more invasive techniques.

Two decades prior to the pioneering MIRPE publication by Nuss et al. (1), reconstructive microsurgery has seen rapid development, primarily for the reconstruction of traumatic loss or oncologic surgery tissue defects. Such microsurgical autologous tissue transplantation techniques, as well as the microvascular sternum turnover $(5,6)$ were sporadically used to repair the congenital anterior thoracic wall defect. Regional or local flaps from the surrounding tissue were found to be suitable only in individual cases since the coincidence of breast hyperplasia and $\mathrm{PE}$ is very rare.

However, the parallel rapid development of MIRPE and microsurgery, as well as endoscopic techniques over the last three decades, triggered an interest in the repair of PE deformities and shifted the indication from purely functional demands to also psychological indications and even more ostensible aesthetic reasons $(7,8)$. The triumphant spread of MIRPE worldwide, supported by electronic media, also renewed interest in alternative and adjunct techniques, not only on the part of physicians but surprisingly also in patients, their parents and a considerable number of adults affected but left untreated for decades. Due to the fact that outstanding techniques like MIRPE cannot always serve as a key for all doors, alternative and adjunct reconstructive or remodeling techniques should be kept in mind. This paper presents established variations and other alternative techniques with ancillary refinements.

\section{Techniques other than MIRPE}

\section{Silastic}

Correction of the funnel depression using alloplastic room temperature vulcanizing (RTV) silastic 382 elastomer commenced half a century ago and was applied predominantly for pronounced cases with physiologically unimpaired cardiopulmonary function. That multiplex material, also used for shunts and insulation of implantable electrodes, is generally well tolerated by human tissue. For funnel chest correction, silastic as a fat-like substance was poured into the funnel epicutaneously. After several minutes, self-vulcanization without any specific catalyst transformed it into a gummy silastic elastomer block similar to a virtual customized mold. Following sterilization and minor chasing, it was implanted in a subcutaneous presternal pocket via an epigastric skin incision and access $(3,4)$.

This elegant and short intervention was performed for an aesthetic indication or because of an explicit severe psychological alteration in adults rather than adolescents. Its advantages are that it is a minimally invasive, lowpriced and brief intervention with a fairly predictable result. The disadvantages of this gummy-like implant are late (after decades) material abrasion and related late seroma formation, potentially with tight calcification, ugly skin bulk and pain. Its application was therefore banned in the United States in the early 1980s. Parallel to developments in aesthetic female breast augmentation and associated improvements in the quality of silicone implant shells and content, funnel chest silastic elastomers have also improved in terms of material composition and tissue compatibility (9). Nowadays, these so-called customized implants are designed at the patient's chest using a mold and the final implant, professionally fabricated elsewhere, is already sterile and ready to be surgically inserted (10). At some centers the required implant can already be designed for the particular patient using CT scan or 3D surface scanner digital processing without intermediate hardware molds (11-13). Similar to breast implants, some surface coatings presumed to minimize capsular contracture are also available. However, in contrast to breast implants with liquid or jelly fillings, capsular contracture of modern silastic flexible implant blocks is unknown to date. The surgical intervention is short, minimally invasive, creates only a small scar remote from the prior deformity (Figures 1-3) and entails low morbidity and very rapid recovery and occasionally might also be performed on a day-hospital basis.

\section{Cartilage chips}

As an alternative to invasive methods and also in order to avoid the burden of alloplastic foreign material, autologous cartilage may serve to correct minor deformities. The method of cartilage shifting is excellently suited to the pectus arcuatum deformity, a rather rare entity consisting of a depression combined with protrusion of individual ribs (Figure $4 A, B$ ). Pectus bars may not work at all and other minor invasive methods may not sufficiently correct such longitudinal sternal wave-like distortion at the manubriogladiolar junction. Furthermore, for various primarily minor asymmetric or for postsurgical deformity residuals at the time of pectus bar explantation, the transfer of cartilage chips is a valuable supplement to finalize the aesthetic appearance (2).

Yet a prerequisite for performing cartilage shifting is existing rib humps with abundant cartilage (14) as a donor site (Figure $4 A$ ). Resected rib portions are processed to small 


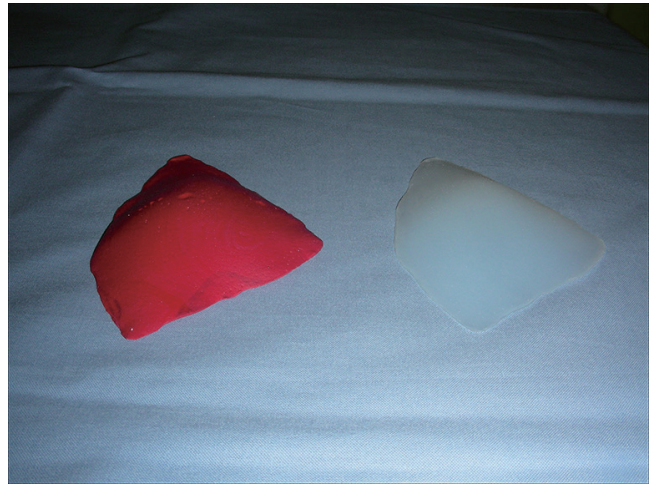

Figure 1 Silastic elastomers. The red one on the left is the hardened moulage; the opaque one on the right is the final implant.

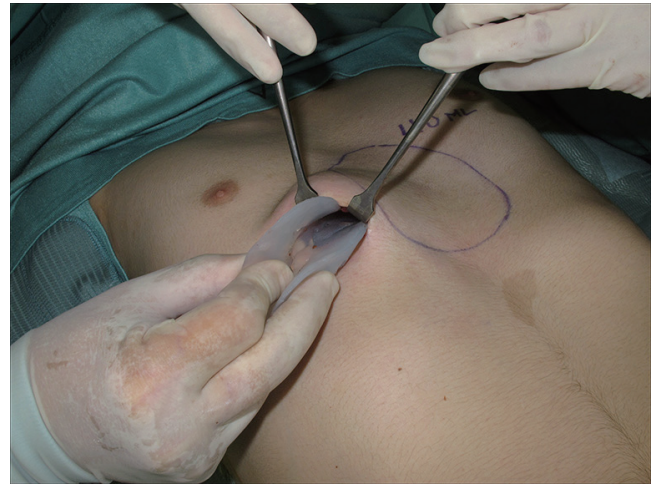

Figure 2 The sterilized silastic is folded and through a small skin incision implanted in a pocket dissected subcutaneously. For aesthetic reasons the incision is made distant from the sternal area.
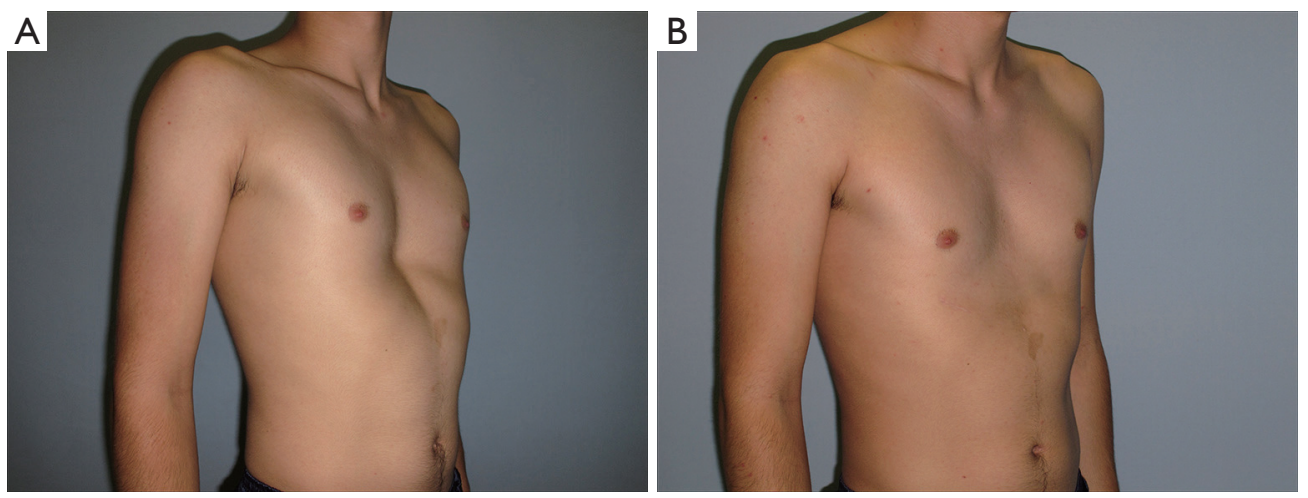

Figure 3 Moderate PE deformity in a 22-year-old patient without functional impairment. (A) Preoperative situation; (B) situation one year thereafter with excellent result and hardly visible scar in the lateral infrapectoral area. PE, pectus excavatum.
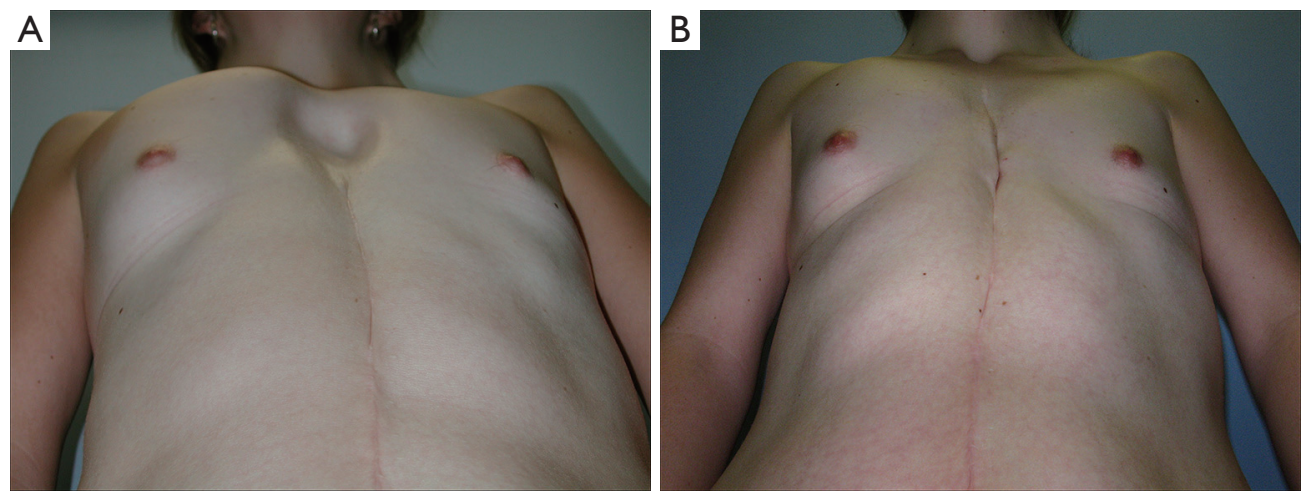

Figure 4 A 20-year-old female with Noonan syndrome and pectus arcuatum deformity. (A) Situation after abdominal wall surgery and prior to rib hump resection; (B) the pectus arcuatum deformity was corrected with cartilage chips shifting from the parasternal rib humps to the presternal depression. 

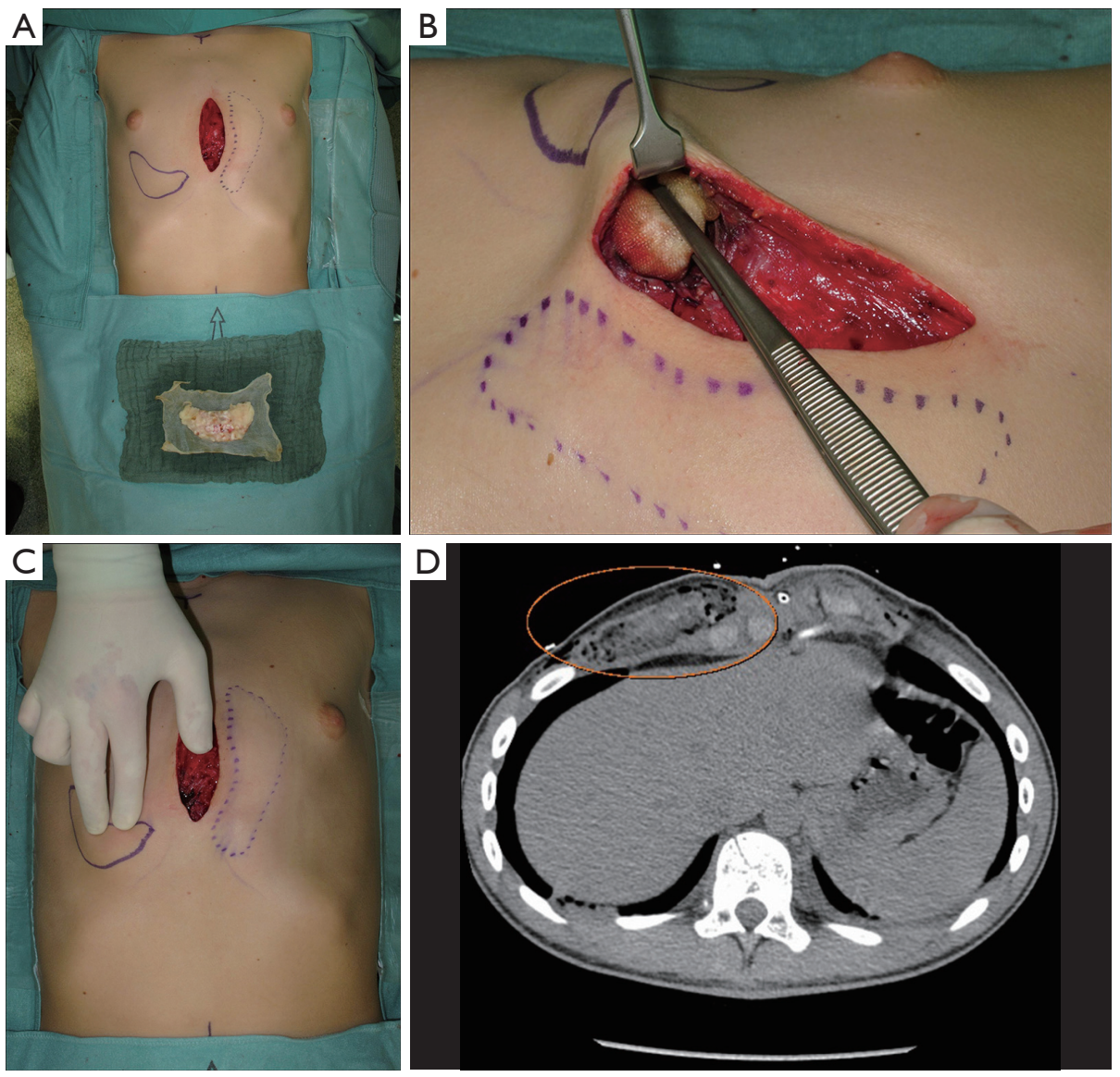

Figure 5 Technique of cartilage chip transfer. (A) Exemplary case with mixed pectus excavatum and carinatum deformity. The resected rib cartilages are comminuted and placed on an absorbable hemostyptic mesh; (B) the cartilage chips are packed and wrapped in the mesh, then implanted in a submuscular pocket at a residual rib depression deformity; (C) the implanted chips are moulded by external manipulation to align the volume bulk with the surrounding skin surface; (D) submuscular (inside orange line) conglomerate of cartilage chips on the right side of the thorax.

chips, wrapped in highly flexible absorbable mesh, and, similar to silastic implants, are embedded in a subcutaneous presternal or epicostal submuscular pocket (Figure $5 A, B$ ). Furthermore, and once implanted, the flexibility of the chips in the absorbable mesh bag allows final shaping and alignment with the surrounding skin surface by digital manipulation (Figure 5C). The rate of resorption is low and once incorporated (Figure 5D) the result lasts for life, in contrast to transplanted fat that changes its volume with body weight.

\section{Lipofilling}

Autologous transfer of fat tissue seems to be a promising technique for primarily filling in a funnel depression without major surgical trauma or scars (15-17). The qualities and characteristics of transplanted autologous fat are well-known, thanks to its widespread application in aesthetic surgery for the treatment of aging skin and subcutaneous tissue. However, heterogeneous behavior of the transplanted fat cells, including stem cells, which depend on their technical treatment and physiological and anatomical conditions at the recipient site, requires ongoing research (18). Congress sessions abound with personal experiences in aesthetic liposhaping of the breast or face, but only a few case reports are available for the presternal area or asymmetric PE $(15,19)$. The failure of this technique to achieve a breakthrough for $\mathrm{PE}$ correction might be due to a general lack of awareness, or limited experience with the technique to date. Presumably, poor results might be due 
to tight adhesion of presternal skin to the bone. Certainly in most cases, typically slim and young PE patients present with a lack of transferable fat and therefore liposhifting is usually not a feasible. It should be reserved for adults with good nutritional status (Figures 6,7A-C).

\section{Microvascular flaps, local flaps}

Autologous transplantation of microvascular flaps for the correction of extensive funnel chest deformities by means of subcutaneous augmentation using vascularized tissue is a rarely used but valuable option. The indication for such time-consuming interventions might be justified when a patient refuses open or minimally invasive thoracic wall remodelling. Abundant tissue suitable for transplantation is more commonly available in adult women than in men. Usually the abdomen or thigh regions provide sufficient

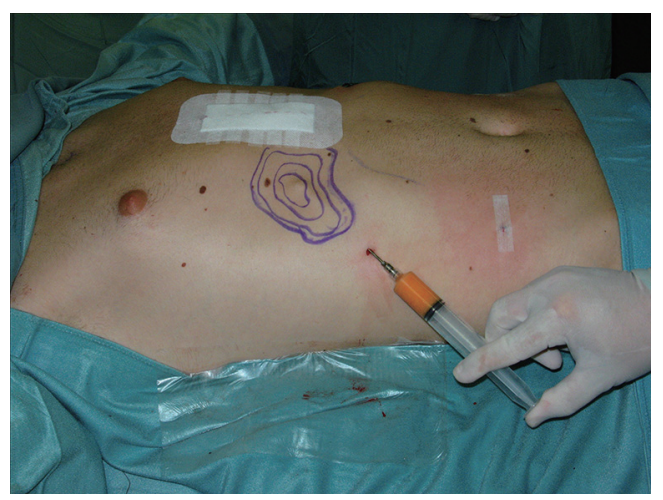

Figure 6 Tissue augmentation at the lateral thoracic wall by autologous lipotransfer, remnant minor depression after keel chest correction. tissue to harvest fasciocutaneous, myocutaneous or adipose flaps, e.g., deep inferior epigastric perforator (DIEP), anterolateral thigh (ALT), transverse myocutaneous gracilis (TMG), or profunda artery perforator (PAP) flaps, for a permanent and long-lasting result similar to oncologic breast reconstruction. However, such procedures require training, skill and experience, and are still prone to microvascular complications in up to about $5 \%$ of patients, even in experienced hands (20-22).

On the other hand, abundant tissue at the female breast might serve as a pedicled flap to be transposed to the funnel depression as a natural filler instead of alloplastic material. Experiences with such flap transplantation and transposition are rare (23) and have taken a back seat due to the rapid development of MIRPE and other thoracoplasty variants. In addition, the treating surgeon must be very familiar with the specific vascular anatomy of the female breast and the techniques of microvascular flap surgery in order to successfully transfer such split breast tissue to the presternal region for an aesthetically pleasing result (Figure 8A-C).

In addition, the previously very promising vascularized sternum turnover, described in a few reports a decade ago, ultimately was not successful $(5,6,24,25)$. The required technical equipment, the complexity of surgery prone to complications (26), as well as time and surgical effort were major contributors to withdrawal from such remodelling in favor of less invasive MIRPE or hybridized techniques.

\section{External tissue expansion with cell seeding}

The vacuum bell after Klobe ${ }^{\circledR}$ has not yet been able to gain significant success as a simple therapy for PE. However, some benefit has been shown with its use as a preparation
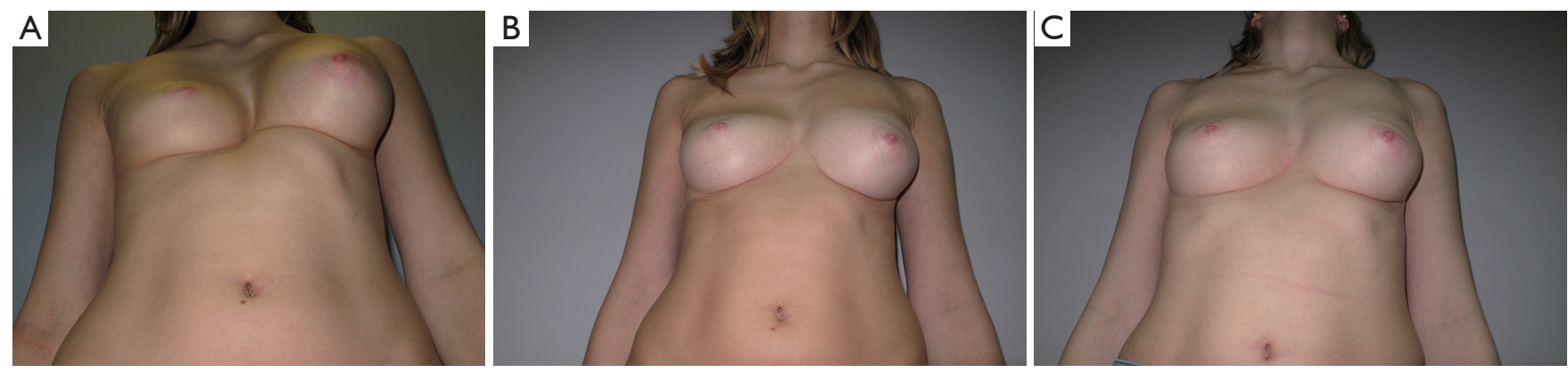

Figure 7 Asymmetric pectus excavatum. (A) Preoperative aspect with malrotation of inclined sternum pretends asymmetry of breasts; (B) residual minor asymmetry of breasts after hybridized MIRPE with right part of thoracic wall considerably but incompletely elevated; (C) distinct improvement after a single intervention with $130 \mathrm{cc}$ lipofilling at both upper quadrants of the right breast. MIRPE, minimally invasive repair of pectus excavatum. 

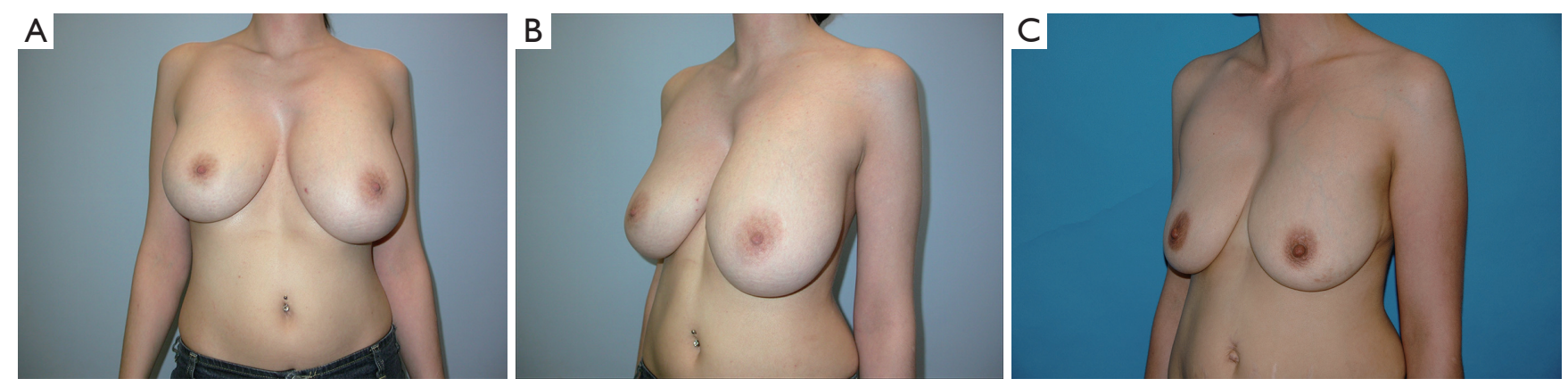

Figure 8 Moderate PE and considerable breast volume asymmetry. (A) This 24-year-old woman primarily refused surgery to her breasts but wanted funnel deformity augmented with silastic. She then accepted unilateral breast reduction for the purpose of transferring the abundant tissue as a pedicled island flap into a subcutaneous presternal pocket; (B) Result eight months postoperatively with the scar from left-side breast reduction, from flap harvest as well as access for preparation of the subcutaneous presernal pocket hidden at the left inframammary crease; (C) result five years postoperatively and three years after pregnancy and breast feeding as well as loss of $10 \%$ of total body weight. PE, pectus excavatum.

for pre- or intraoperative auxiliary treatment $(27,28)$. Another very promising technique is the BRAVA $^{\circledR}$ (breast enhancement and shaping system), which was designed for breast reconstruction or augmentation and works as an external tissue expander to produce edema and increase blood perfusion (29-31). This procedure creates a socalled endogenous organic scaffold that can take fat grafts by lipofilling much better than untreated tissue can. As a future potential alternative, such lipofilling procedures, similar to BRAVA $^{\circledR}$ in that they are minimally invasive and produce only minimal scars while utilizing the Klobe ${ }^{\circledR}$ bell in the presternal region, can definitely be advantageous and attractive options.

\section{Macrolane}

Hyaluronic acid, applied millions of times in aesthetic medicine for the treatment of wrinkles in the aging face, also found its way to physicians who occasionally use it to fill in a PE deformity (32). Apart from the prohibitive price due to the large volume needed to induce a visible improvement, the effect is only temporary due to resorption and the funnel will relapse to its original extent one to two years after injection. To justify its application, one might argue that it is a minimally invasive simulation of a result that might be surgically attainable at a later time.

\section{Tissue engineering}

Correction of minor PE deformities primarily or secondarily using injection transplantation of cultured autologous chondrocytes is an attractive further development of cartilage chips transfer. As sufficient amounts of chips are only available from considerable deformities with abundant cartilage humps, de novo generation of cartilage or fat tissue for filling in funnels may be options when resection is neither feasible nor necessary. Autologous cell suspensions or seeded scaffolds such as for tissue engineering are a matter of ongoing research and controlled clinical trials, currently a special topic in breast augmentation surgery (31,33).

\section{Special aspects in female patients}

Adolescent patients with ostensible breast hypoplasia occasionally also suffer from PE, but the complex deformity is often misunderstood as an exclusively breast tissue developmental problem. Usually in such cases, the breasts are distorted medially, causing so-called strabism of the breasts and giving the illusion of hypoplasia merely by their depressed position (Figure 9). This might be the case for symmetric as well as asymmetric deformities (Figure $7 A$ ). A recent study (34) confirmed that breast volume in most PE patients with asymmetry does not differ between the right and left breast, but might be adjusted to provide a symmetric appearance through thoracic wall remodelling, without the need for autologous or alloplastic breast augmentation $(8,35)$. Breast tissue volume very rarely develops asymmetrically, but in such patients, variable expression of a congenital deformity known as Poland 
syndrome must be considered.

However, some of these patients are then usually recommended to have their breasts corrected by implant or lipofilling augmentation only. Such simple correction attempts, however, neglect the underlying thoracic wall deformity for the sake of hasty superficial cosmetic surgery, which may lead to an unfavorable result (Figure 10A,B). The deformity usually is caused by a complex deficit of the anterior thoracic wall, not of the female breast tissue itself $(8,34,35)$. The aspect of a depressed sternum thus cannot be primarily corrected by augmentation of unaffected neighboring breast tissue, because such augmentation may even enhance the optical depth of the funnel as well as the strabism distortion of the breasts (Figures 9,11A,B). On the contrary, temporary implantation of a pectus bar with the scars hidden in the inframammary crease and

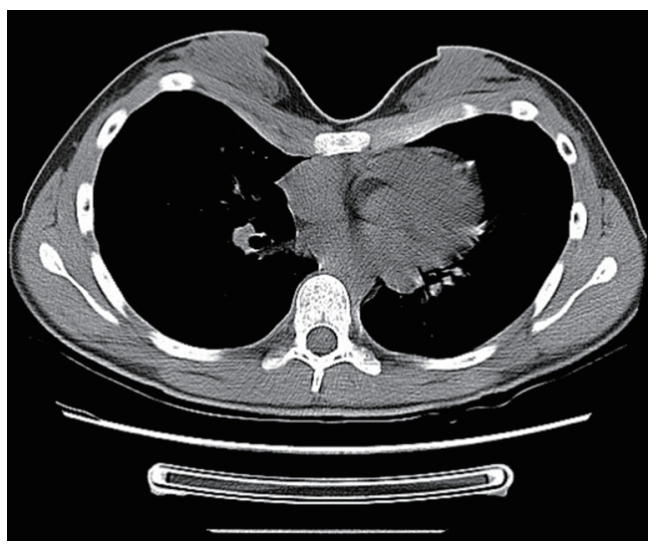

Figure 9 Asymmetric funnel deformity giving the illusion of breast hypoplasia and causing strabism of the nipple areola complexes. without surgery of the female breast (Figure 11C,D) itself is much more advantageous in the long run (Figure 12A,B). Despite its common use for breast augmentation and the rapid development of the quality and durability of material, permanent breast implants still demonstrate late complications such as capsular contraction, implant rupture, late infection and shape distortion, with the need for continuing surgery after years or decades. However, without such problems, implants or lipofilling can definitely provide very pleasant and aesthetically perfect results, thus justifying their place in the armamentarium of breast and chest wall deformities as long as the depressed sternum is only has a minor inclination and breast hypoplasia is the main aesthetic complaint. In the corrected asymmetric PE deformity, a unilateral breast implant may occasionally be necessary to achieve symmetry (Figure 13A-C), or bilateral augmentation may be performed as an additional aesthetic intervention (Figure 14A,B).

Customized silastic devices and lipofilling may also be considered as an adjunct procedure in asymmetric cases, being particularly suitable in female patients for the correction of minor postsurgical PE deformities (Figure $7 B, C$ ).

Selection of reconstructive or remodeling options offered to a female patient must be based on a distinct analysis of the underlying problem and cause of breast distortion or asymmetry (Figure 9). The decision-making process must also factor in the patient's expectations, with comprehensive informed consent concerning the pros and cons of the various treatment approaches and the objective results that can be achieved. Particularly during puberty, female patients develop their specific gender appearance in a psychologically delicate phase and therefore, apart from
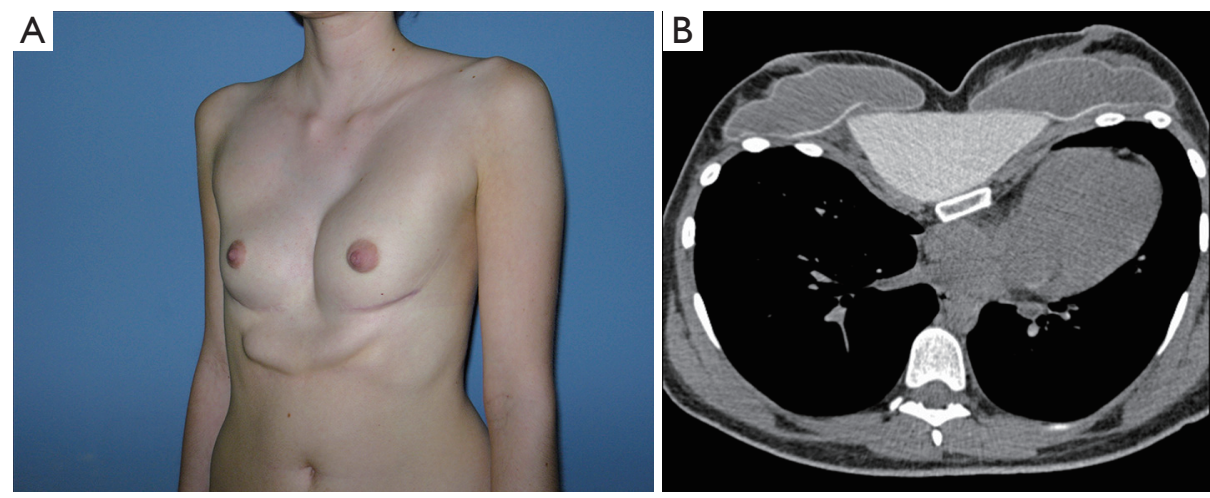

Figure 10 Undesirable complication. (A) The PE deformity was treated by implanting two breast prostheses and a large silastic block, causing a severe feeling of unpleasant pressure especially when in a supine position; (B) CT scan of same patient showing the alloplastic volume that detracts from the patient's quality of life. PE, pectus excavatum. 

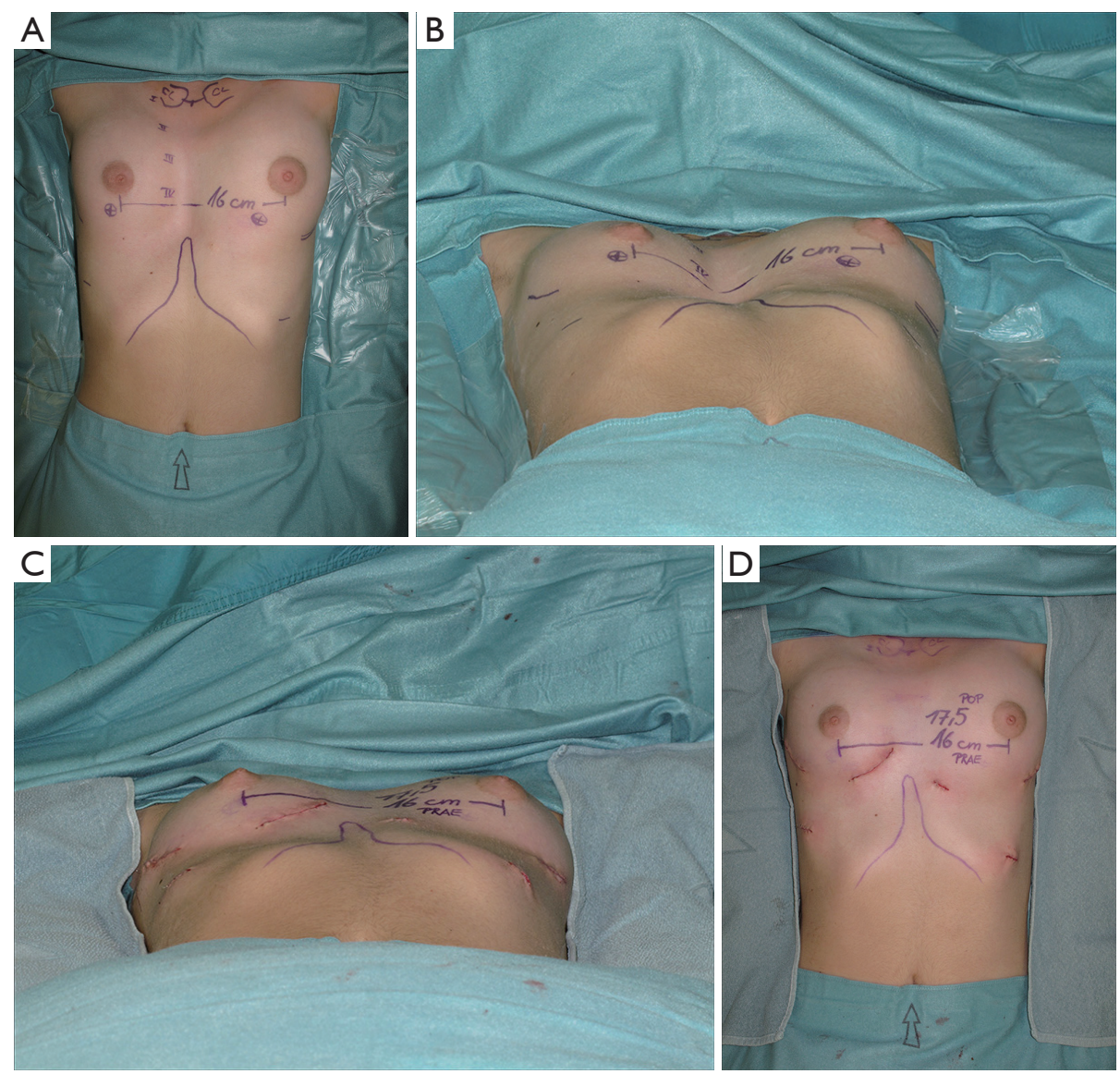

Figure 11 Asymmetric distortion of breasts conjoined with PE deformity. (A,B) The underlying chest wall deformity causes appearance of breast strabism; (C,D) intraoperative situs after hybridized MIRPE (MOVARPE) only. Notice the increase of $1.5 \mathrm{~cm}$ in intermamillary distance; strabism and asymmetry corrected without silicone implants. MIRPE, minimally invasive repair of pectus excavatum.
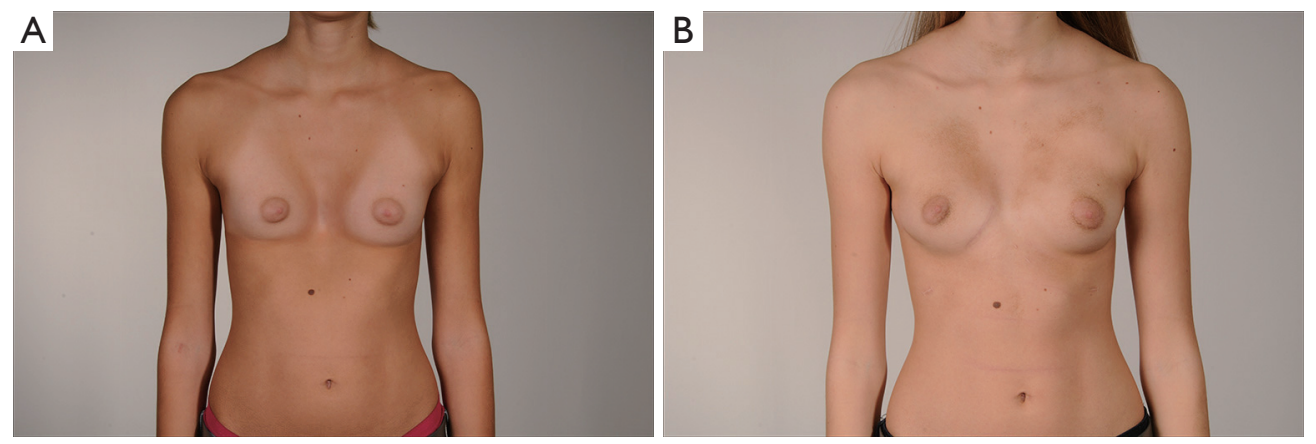

Figure 12 Another patient with asymmetric PE (A) but same correction as in patient in Figure 11. Postoperative appearance one year after pectus bar removal and three years after initial PE repair (B) without any additional refinement surgery. PE, pectus excavatum. 

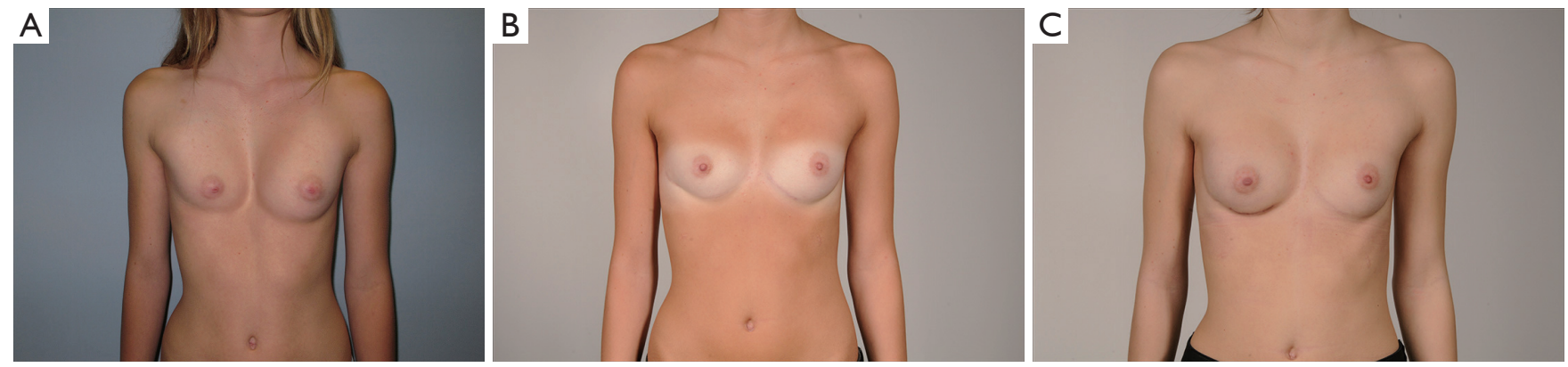

Figure 13 Another patient with asymmetric PE (A) but same initial correction as in patient in Figures 11 and 12 . Postoperative appearance two years after initial PE repair prior to pectus bar removal (B). Situation six months after pectus bar removal and simultaneous correction with a $150 \mathrm{cc}$ breast implant on the right side (C). PE, pectus excavatum.
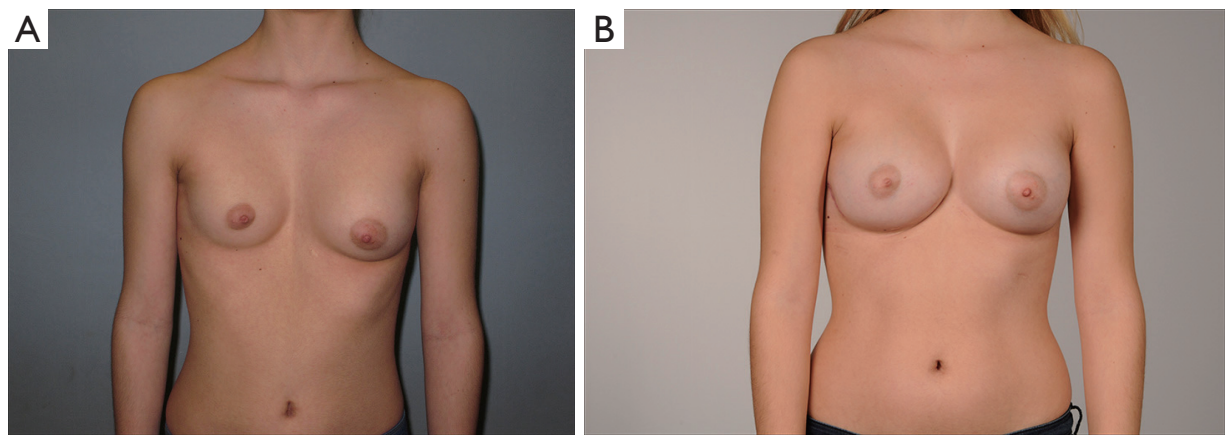

Figure 14 PE asymmetry and uncommon asymmetric breast volumes. (A) In addition, minimal tuberous breast deformity on the left side; (B) MOVARPE correction did not produce sufficient symmetry. Final result after pectus bar removal and breast augmentation with 270 cc implant on the right and $160 \mathrm{cc}$ implant on the left side. PE, pectus excavatum.

cases with considerable functional impairment, timing of corrective surgery at the thoracic wall should consider breast tissue growth as well as somatic and mental maturation $(8,36)$. Finally and particularly for young female patients, skin incisions should be placed along the relaxed skin tension lines (RSTL) in order to minimize the appearance of scars.

\section{Perspectives}

Somatic disorders reported by patients and therefore subjectively perceived are often overrated, in order to force a medical indication for surgical correction to be covered by a compulsory insurance company. Such non-specific symptoms such as palpitations, thoracic wall pain, dyspnea, angina pectoris or exercise intolerance may be fictitious or extracted from various media and internet sources. These fictitious symptoms have to be distinguished from objective functional or structural cardiorespiratory impairment by means of specific cardiorespiratory examination or various thoracic indices. This is essential for establishing the indication for and selecting an appropriate PE repair technique.

However, the psychological alterations caused by a more or less pronounced deformity can also be significant, ranging from normal to even suicidal cases with severely restricted quality of life. Therefore, in patients with no somatic symptoms and with proven normal cardiorespiratory function, it also appears to be important to examine the patient's mental status, regardless of how severe the deformity is. Although a patient's psychological burden is not easily measurable, it requires time-consuming psychological and specific but standardized testing by specialists. Such tests are specially designed for patients suffering from anterior wall deformities $(7,36,37)$. Under certain circumstances, specific persons, whose psychological alterations or psychosocial deficits are not attributable to 
the PE deformity can be isolated and might benefit from psychotherapy only. Potentially, PE patients might require psychotherapy in addition to surgical treatment, namely prior to it, parallel to it or thereafter.

For patients with diagnostic psychological findings and minor functional impairments that do not yet justify invasive surgery, minor corrective procedures can be offered. Such minor surgery may be possible solely for the purpose of improving the aesthetic appearance of the deformity to an extent that improves quality of life subjectively and objectively.

Since existing modern or standard techniques are not yet able to produce perfect results in every case, especially in cases of asymmetry, adjuvant plastic surgery methods can improve the final results. They may not eliminate the need for thoracic wall remodelling, but can serve as solitary or ancillary methods of extrathoracic remodeling or aesthetic refinement of minor or remnant defects. It would therefore be desirable for specialists of various disciplines to come together for interdisciplinary treatment, or for all treating physicians to become familiar with all common techniques for the best achievable treatment of the patients entrusted to our care.

Nowadays, more than ever, plastic surgeons are called upon and have the duty to not only recognize their own limits, but to also set boundaries for their patients concerning the feasibility of an intended intervention in light of potentially unrealistic aesthetic expectations. In daily practice, this means that the patient must be informed that although a deformity may be improved, the result may not always be perfect. Just because a new method or technique has become available does not mean it should be used in every possible case. Bide your time, reexamine the indication and take a cautious approach to different available methods. After all, this is sometimes of paramount importance in separating suitable patients from those with unfulfillable expectations.

\section{Conclusions}

The asymmetric and extensive cases of $\mathrm{PE}$ still require a highly individualized approach with surgical standard procedures and their subset of modifications $(38,39)$ as well as the development of ongoing refinements like autologous tissue transfer as single, adjunct or secondary interventions. However, in cases of symmetric and mild-to-moderately deformed PE, MIRPE is nowadays the established gold standard for child and adolescent patients, although other techniques might also serve well in adults. Moreover, the correction of all varieties of $\mathrm{PE}$ deformities no longer seems to be restricted to single specialities. Instead, the diversity of corrective techniques is driving an increasingly multidisciplinary approach, which advocates that correction of such PE deformities remain at comprehensive care centers with high patient turnover and multimodal facilities and experience.

\section{Acknowledgements}

None.

\section{Footnote}

Conflicts of Interest: The author has no conflicts of interest to declare.

\section{References}

1. Nuss D, Kelly RE Jr, Croitoru DP, et al. A 10-year review of a minimally invasive technique for the correction of pectus excavatum. J Pediatr Surg 1998;33:545-52.

2. Del Frari B, Schwabegger AH. Diced autologous rib cartilage for primary treatment or refinement of minor chest wall deformities. Plast Reconstr Surg 2011;128:154-62.

3. Bailey BN. Pectus excavatum: masking with silastic RTV 382 vulcanised in situ. Br J Plast Surg 1977;30:227-30.

4. Bruck JC, Hörtnagl H, Bauer M, et al. A fresh approach in the treatment of pectus excavatum. Chir Plast 1982;6:263-74.

5. Ninkovic M, Schwabegger A, Gardetto A, et al. Free sternum turnover flap for correction of pectus excavatum deformity. Plast Reconstr Surg 2003;112:1355-61.

6. Liu T, Liu H, Li Y. Comparison of the Nuss and sternal turnover procedures for primary repair of pectus excavatum. Asian J Surg 2014;37:30-4.

7. Kelly RE Jr, Cash TF, Shamberger RC, et al. Surgical repair of pectus excavatum markedly improves body image and perceived ability for physical activity: multicenter study. Pediatrics 2008;122:1218-22.

8. Schwabegger AH, Del Frari B, Pierer G. Aesthetic improvement of the female breast in funnel chest deformity by surgical repair of the thoracic wall: indication or lifestyle surgery? Plast Reconstr Surg 2012;130:245e-253e.

9. Schwabegger AH, Del Frari B. Custom-made silicone implants. In: Schwabegger AH. editor. Congenital thoracic wall deformities: Diagnosis, Therapy and Current 
Developments. New York: Springer, 2011:143-53.

10. Saour S, Shaaban H, McPhail J, et al. Customised silicone prostheses for the reconstruction of chest wall defects: technique of manufacture and final outcome. J Plast Reconstr Aesthet Surg 2008;61:1205-9.

11. Johnson PE. Refining silicone implant correction of pectus excavatum through computed tomography. Plast Reconstr Surg 1996;97:445-9.

12. Kovacs L, Yassouridis A, Zimmermann A, et al. Optimization of 3-dimensional imaging of the breast region with 3-dimensional laser scanners. Ann Plast Surg 2006;56:229-36.

13. Kovacs L, Eder M, Brossmann C, et al. Thoracic wall deformities: 3-D scanning and computerized remodeling. In: Schwabegger AH. editor. Congenital thoracic wall deformities: Diagnosis, Therapy and Current Developments. New York: Springer, 2011:307-17.

14. Park CH, Kim TH, Haam SJ, et al. Rib overgrowth may be a contributing factor for pectus excavatum: Evaluation of prepubertal patients younger than 10years old. J Pediatr Surg 2015;50:1945-8.

15. Mattesich M, Schwabegger AH. Lipofilling for funnel chest and similar or adjacent thoracic wall deformities. In: Schwabegger AH. editor. Congenital thoracic wall deformities: Diagnosis, Therapy and Current Developments. New York: Springer, 2011:159-63.

16. Coleman SR. Long-term survival of fat transplants: controlled demonstrations. Aesthetic Plast Surg 1995; 19:421-5.

17. Iannace C, Di Libero L, Manetta F, et al. Coleman lipofilling: experience of an Italian group and review of the literature. Chir Ital 2009;61:67-75.

18. Ho Quoc C, Taupin T, Guérin N, et al. Volumetric evaluation of fat resorption after breast lipofilling. Ann Chir Plast Esthet 2015;60:495-9.

19. Pereira LH, Sterodimas A. Free fat transplantation for the aesthetic correction of mild pectus excavatum. Aesthetic Plast Surg 2008;32:393-6.

20. Las DE, de Jong T, Zuidam JM, et al. Identification of independent risk factors for flap failure: A retrospective analysis of 1530 free flaps for breast, head and neck and extremity reconstruction. J Plast Reconstr Aesthet Surg 2016;69:894-906.

21. Michlits W, Windhofer C, Papp C. Pectus excavatum and free fasciocutaneous infragluteal flap: a new technique for the correction of congenital asymptomatic chest wall deformities in adults. Plast Reconstr Surg 2009;124:1520-8.
22. Raab N, Holm-Muehlbauer C, Ninkovic M. Free superficial inferior epigastric artery flap for aesthetic correction of mild pectus excavatum. Plast Reconstr Surg 2009;123:209e-11e.

23. Schwabegger AH, Piza-Katzer H, Pauzenberger R, et al. The internal mammary artery perforator (IMAP) breastflap harvested from an asymmetric hyperplastic breast for correction of a mild funnel chest deformity. Aesthetic Plast Surg 2011;35:928-32.

24. Iida H, Sunazawa T, Ishida K, et al. Surgical repair of pectus excavatum not requiring exogenous implants in 113 patients. Eur J Cardiothorac Surg 2010;37:316-21.

25. Tang Chen YB, Chen JS, Lee YC, et al. Revascularization of turnover sternum: A definitive treatment for intractable funnel chest. Microsurgery 1999;19:296-302.

26. Schwabegger AH, Piza H. Free sternum turnover flap for correction of pectus excavatum deformity. Plast Reconstr Surg 2005;116:1182.

27. Schier F, Bahr M, Klobe E. The vacuum chest wall lifter: an innovative, nonsurgical addition to the management of pectus excavatum. J Pediatr Surg 2005;40:496-500.

28. Haecker FM. The vacuum bell for conservative treatment of pectus excavatum: the Basle experience. Pediatr Surg Int 2011;27:623-7.

29. Khouri RK, Rigotti G, Khouri RK Jr, et al. Tissueengineered breast reconstruction with Brava-assisted fat grafting: a 7-year, 488-patient, multicenter experience. Plast Reconstr Surg 2015;135:643-58.

30. Ho Quoc C, Piat JM, Carrabin N, et al. Breast reconstruction with fat grafting and BRAVA(®) preexpansion: Efficacy evaluation in 45 cases. Ann Chir Plast Esthet 2016;61:183-9.

31. Kosowski TR, Rigotti G, Khouri RK. Tissue-Engineered Autologous Breast Regeneration with Brava ${ }^{\circledR}$-Assisted Fat Grafting. Clin Plast Surg 2015;42:325-37, viii.

32. Sinna R, Perignon D, Assaf N, et al. Use of Macrolane to treat pectus excavatum. Ann Thorac Surg 2012;93:e17-8.

33. Tamai M, Nagasao T, Yanaga H, et al. Correction of secondary deformity after Nuss procedure for pectus excavatum by means of cultured autologous cartilage cell injection. Int J Surg Case Rep 2015;15:70-3.

34. Rha EY, Kim JM, Yoo G, et al. Is breast asymmetry caused by volume differences in women with pectus excavatum? J Plast Reconstr Aesthet Surg 2016;69:470-4.

35. Fonkalsrud EW. Management of pectus chest deformities in female patients. Am J Surg 2004;187:192-7.

36. Rumpold G, Lair M. Psychological investigation. Psychosocial effects of a funnel or keel breast deformity. 
In: Schwabegger AH. editor. Congenital Thoracic Wall Deformities: Diagnosis, Therapy and Current Developments. New York: Springer, 2011:79-82.

37. Cook SA, Rosser R, Salmon P. Is cosmetic surgery an effective psychotherapeutic intervention? A systematic review of the evidence. J Plast Reconstr Aesthet Surg 2006;59:1133-51.

38. Schwabegger AH. Surgical technique with the modified hybrid access in adolescents and adults, the MOVARPE

Cite this article as: Schwabegger AH. Pectus excavatum repair from a plastic surgeon's perspective. Ann Cardiothorac Surg 2016;5(5):501-512. doi: 10.21037/acs.2016.09.01 (minor open videoendoscopically assisted repair of pectus excavatum) technique. In: Schwabegger AH. editor. Congenital Thoracic Wall Deformities: Diagnosis, Therapy and Current Developments. New York: Springer, 2011:132-42.

39. Del Frari B, Schwabegger AH. Clinical results and patient satisfaction after pectus excavatum repair using the MIRPE and MOVARPE technique in adults: 10-year experience. Plast Reconstr Surg 2013;132:1591-602. 